\title{
Nonlinear Distributed Model for Bulk Acoustic Wave Resonators
}

\author{
Carlos Collado, Member, IEEE, Eduard Rocas, Student Member, IEEE, Jordi Mateu, Member, IEEE, \\ Alberto Padilla, Student Member, IEEE, and Juan M. O'Callaghan, Senior Member, IEEE
}

\begin{abstract}
This work expands the model proposed by Krimtholz, Leedom, and Matthaei (KLM) model to account for the nonlinear effects occurring in acoustic devices due to the nonlinear stiffened elasticity. We show that a nonlinear distributed capacitance in the acoustic transmission line of the KLM model can account for the distributed nature of the nonlinear effects. Specifically, we use the nonlinear telegrapher's equation to find closed-form equations for intermodulation distortion and harmonic generation. We confirm the validity of these equations by comparing their results with those provided by a KLM equivalent circuit in which the nonlinear transmission line is implemented by cascading many $L-C$ cells having a voltage-dependent capacitance. To further confirm the model, we show measured nonlinear effects in a thin film bulk acoustic resonator in close agreement with the equivalent circuit simulations.
\end{abstract}

Index Terms-Bulk acoustic wave (BAW), film bulk acoustic resonator, harmonic generation, intermodulation distortion, nonlinear Krimtholz, Leedom, and Matthaei (KLM), nonlinear stiffened elasticity, nonlinearities.

\section{INTRODUCTION}

B ULK ACOUSTIC WAVE (BAW) technology is capable of producing miniature high $Q$ resonators, which are essential elements in compact filters having low-insertion loss and high-frequency selectivity. A widespread use of this technology is expected in the ever-growing wireless market, where handheld devices need to accommodate for requirements such as spectrum crowding, high bandwidth demand, miniaturization, and low cost [1].

However, there are still limitations that may exclude the use of BAW resonators in some microwave applications. In particular, their inherent nonlinear behavior [2] may cause intermodulation distortion (IMD), harmonic generation, and detuning and/or saturation of the filter frequency response.

Manuscript received December 29, 2008; revised August 26, 2009. First published November 13, 2009; current version published December 09, 2009. This work was supported in part by the Spanish Government (CICYT) under Grant TEC-2006-13248-C04-02/TCM.

C. Collado, E. Rocas, A. Padilla, and J. M. O'Callaghan are with the Department of Signal Theory and Communications, Universitat Politècnica de Catalunya (UPC), Barcelona 08034, Spain (e-mail: collado@tsc.upc.edu; eduard.rocas@tsc.upc.edu; alberto.padilla@tsc.upc.edu; joano@tsc.upc.edu).

J. Mateu is with the Department of Signal Theory and Communications, Universitat Politècnica de Catalunya (UPC), Barcelona 08034, Spain and also with the Centre Tecnològic de Telecomunicacions de Catalunya (CTTC), Castelledefels, Barcelona 08860, Spain (e-mail: jmateu@tsc.upc.edu).

Color versions of one or more of the figures in this paper are available online at http://ieeexplore.ieee.org.

Digital Object Identifier 10.1109/TMTT.2009.2034211
Although the nonlinear effects may be due to several causes, most previous publications point to thermal effects and the nonlinearity in stiffness, piezoelectric coefficient, and permittivity as the dominant ones [3]. Quantifying such nonlinear effects with material parameters such as the nonlinear stiffened elasticity is crucial to fully understand the nonlinear behavior of BAW resonators. On the other hand, equivalent circuits of BAW resonators are needed to predict the nonlinear effects occurring in more complex devices, such as filters with several resonators. A definition of the equivalent circuit elements (such as a voltage-dependent capacity) consistent with the material parameters (such as the nonlinear stiffened elasticity) would be very useful to relate material properties with the final system performance.

Tiersten [4] reported on a nonlinear lumped equivalent circuit for rotated Y-cut quartz resonators and its corresponding closed-form expressions to predict the third-order IMD. Thereafter, other works [5]-[9] used lumped approaches based in Tiersten's equations or in nonlinear versions of the Butterworthvan-Dike equivalent circuit (BVD) [10] in which one or several lumped elements of the acoustic branch (series $R-L-C$ circuit) are nonlinear. Some of our previous work [11] also uses a phenomenological approach based on the BVD circuit to model the IMD occurring in a film bulk acoustic resonator (FBAR).

These different approaches based on lumped element equivalent circuits are simple and useful for modeling some limited manifestations of the nonlinear effects. However, the values of the lumped elements in these equivalent circuits are jointly affected by material parameters and device-specific parameters, such as resonator size and geometry. In these conditions, it is hard to consistently relate the values of equivalent circuit elements to material parameters.

A distributed model can be found in [12], in which the authors extend Mason's linear circuit to the nonlinear region. In this paper, we address this problem by extending our preliminary work in [13], which proposes a distributed nonlinear equivalent circuit based on the lineal model proposed by Krimtholz, Leedom, and Matthaei (KLM) [14]. As stated in [14], the roles of the mechanical and electrical parts of the circuit are more clearly distinguished in the KLM equivalent circuit than in the Mason's one, which simplifies the nonlinear extension.

Our version of equivalent circuit is made by replacing the acoustic line in the KLM model with many cascaded elemental $L-C$ cells. These cells have nonlinear elements that are consistent with the stress-strain curve of the piezoelectric layer in the BAW resonator. With this model we are able to relate the nonlinearities in the equivalent circuit (specifically a voltage-depen- 
dent distributed capacitance in a transmission line) with nonlinearities in the material (stiffened elasticity).

An additional advantage of the model we are proposing is its validity over wide frequency ranges, which makes the model valid for predicting the generation of second harmonics and out-of-band intermodulation products. This is unlike the simpler version of the BVD model, which is limited to a narrow frequency range if no additional acoustic branches are considered.

As an example, we use the equivalent circuit to fit measured linear and nonlinear data in a thin film bulk acoustic resonator. Furthermore, we use the equivalent circuit to derive closed-form equations for some of the most relevant nonlinear effects: intermodulation distortion and second harmonic generation.

\section{NONLINEAR ACOUSTIC TRANSMisSiOn LinE MODEL}

As mentioned above, the proposed equivalent circuit is based on an extension of the linear KLM model to account for the distributed nonlinear effects in the acoustic wave. This section reviews the basic concepts of the KLM model and details the additional considerations used to include the nonlinear effects in the model.

\section{A. Linear KLM Model}

The conventional KLM model [14] (see Fig. 1) includes a transmission line that accounts for the usual equivalences in acoustic wave devices (voltage $v$ is equivalent to force $F$ and current $i$ to velocity). The characteristic impedance $Z_{0}$ and phase velocity $v_{p}$ in this transmission line are given by

$$
\begin{aligned}
& Z_{0}=\rho v_{p} A \\
& v_{p}=\sqrt{\frac{c^{D}}{\rho}}
\end{aligned}
$$

where $A$ and $\rho$ are, respectively, the area of the electrodes and the density of the piezoelectric material. The term $c^{D}$ represents the stiffened elasticity and is obtained from the constitutive equations describing the stress-strain curve in a piezoelectric material [15]. Its value is equal to the elasticity under constant electric field $c^{E}$ plus an additional term that depends on the piezoelectric constant $e$ and the dielectric constant $\varepsilon^{S}$ as

$$
c^{D}=c^{E}+\frac{e^{2}}{\varepsilon^{S}}
$$

The transformation ratio $\phi$, electrical capacity $C_{0}$, and reactance $X_{1}$ shown in Fig. 1 are given by

$$
\begin{aligned}
\phi & =\frac{\omega Z_{0}}{2 h} \frac{1}{\sin \left(\frac{\omega l}{2 v_{p}}\right)} \\
C_{0} & =\varepsilon^{S} \frac{A}{l} \\
X_{1} & =\frac{h^{2}}{\omega^{2} Z_{0}} \sin \left(\frac{\omega l}{v_{p}}\right)
\end{aligned}
$$

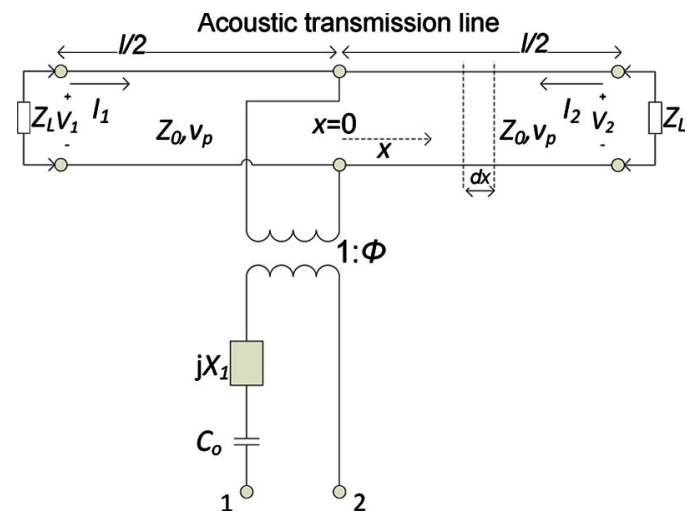

Fig. 1. Equivalent KLM circuit of a BAW resonator [14]. The acoustic transmission line of length $l$ extends along the $x$-axis and is connected to an electrical network at $x=0$. Ports 1 and 2 are the input and output ports.

where $h$ is a piezoelectric constant of the crystal $\left(h=e / \epsilon^{S}\right)$ and $l$ is its thickness.

The telegrapher's equations of the equivalent transmission line [15] for an acoustic wave propagating along the $x$-axis can be written as

$$
\begin{aligned}
& \frac{\partial i}{\partial x}=-\frac{\partial\left[C_{d} v\right]}{\partial t} \\
& \frac{\partial v}{\partial x}=-\frac{\partial\left[L_{d} i\right]}{\partial t}
\end{aligned}
$$

being $C_{d}=1 /\left(A \cdot c^{D}\right)$ the distributed capacitance and $L_{d}=$ $\rho \cdot A$ the distributed inductance.

\section{B. Nonlinear KLM Model}

Although it is supposed that the elasticity, which relates the stress with the strain at constant electric field, is the main contribution to the nonlinear response [16], there are also other sources that may be considered, such as the piezoelectric constant or the permittivity [3]. These sources may be drawn together in a unique term $c^{D}$ in (3) which depends on the stress $T$ as

$$
c^{D}(T)=c_{0}^{D}+\Delta c_{N L}^{D}(T)
$$

This function models the piezoelectric stress-strain curve, which for weak nonlinearities can be expanded in a Taylor's series as

$$
c^{D}(T)=c_{0}^{D}+\Delta c_{1}^{D} \cdot T+\Delta c_{2}^{D} \cdot T^{2}+\cdots
$$

where $\Delta c_{1}^{D}, \Delta c_{2}^{D}, \ldots$ account for the strength of the nonlinear effects.

In the KLM model, the voltage is equivalent to the force, which is uniform in a cross section of the acoustic transmission line for the propagating mode of interest. Therefore, we can scale the independent variable $T$ in $c^{D}(T)$ with the area $A$ using $F=-A T[15]$. We can then denote $c^{D}(F)$

$$
c^{D}(F)=c_{0}^{D}-\frac{\Delta c_{1}^{D}}{A} \cdot F+\frac{\Delta c_{2}^{D}}{A^{2}} \cdot F^{2}+\cdots
$$


Since, in the equivalent circuit, the distributed capacitance in the transmission line $\left(C_{d}\right)$ depends on $c^{D}$ through $C_{d}=1 /(A$. $\left.c^{D}\right), c^{D}(F)$ can be modeled by a nonlinear capacitance

$$
C_{d}(v)=C_{d, 0}+\Delta C_{N L}(v)
$$

where $C_{d, 0}$ represents the linear term of the distributed capacitance. The additional nonlinear term can also be expanded in a Taylor's series as $\Delta C_{N L}(v)=\Delta C_{1} v+\Delta C_{2} v^{2}+\cdots$, where, for example, the first two terms $\Delta C_{1}$ and $\Delta C_{2}$ can be written as

$$
\begin{aligned}
& \Delta C_{1}=\frac{1}{A c_{0}^{D}} \frac{\Delta c_{1}^{D}}{A c_{0}^{D}} \\
& \Delta C_{2}=\frac{1}{A c_{0}^{D}}\left(-\frac{\Delta c_{2}^{D}}{A^{2} c_{0}^{D}}+\left(\frac{\Delta c_{1}^{D}}{A c_{0}^{D}}\right)^{2}\right) .
\end{aligned}
$$

Note that (13) and (14) relate the device-independent nonlinear terms of the piezoelectric constants $\Delta c_{1}^{D}$ and $\Delta c_{2}^{D}$ with the nonlinear terms of the equivalent circuit $\Delta C_{1}$ and $\Delta C_{2}$.

Using the nonlinear distributed capacitance (12) into the telegrapher's (7) and (8), we obtain

$$
\begin{aligned}
& \frac{\partial v}{\partial x}=-L_{d} \frac{\partial i}{\partial t} \\
& \frac{\partial i}{\partial x}=-C_{d, 0} \frac{\partial v}{\partial t}-\frac{\partial i_{n l}}{\partial x}
\end{aligned}
$$

with

$$
\frac{\partial i_{n l}}{\partial x}=\frac{\partial}{\partial t}\left(\Delta C_{N L}(v) v\right) .
$$

Equations (15)-(17) describe the nonlinear behavior of an acoustic transmission line and will be used in Section III to derive closed-form expressions that relate nonlinear measured IMD and harmonics with the stiffened elasticity of the piezoelectric layer.

Before going into details on the formulation, we summarize the assumptions considered throughout the paper.

1) The equivalent circuit of an elemental section of an acoustic transmission line used in this work is outlined in Fig. 2. For simplicity, we do not consider the series resistance and shunted conductance that would model the losses in an acoustic transmission line [15], however note that those elements could be easily included in the electrical model of Fig. 2.

2) Other sources of nonlinearities beyond those included in the definition of $c^{D}$ in (3), such as self-heating effects, changes in the density, or nonlinear friction effects are not considered. If necessary, the model could be easily expanded with a nonlinear distributed inductance, series resistance, or shunted conductance.

3) We are not taking into account the nonlinearities arising from $C_{0}$ nor those arising from $X_{1}$ and $\phi(4)-(6)$. We assume that the nonlinearity in $C_{0}$ due to $\varepsilon^{S}$ and the changes in $v_{p}$ and $h$ with the stress are negligible compared with those arising inside the resonator due to the nonlinear stiffened elasticity.

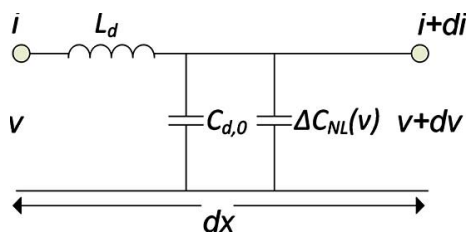

Fig. 2. Lossless transmission line cell modeling and infinitesimal length section of the acoustic transmission line.

\section{CLOSED-FORM EXPRESSIONS FOR IMD AND SECOND HARMONIC}

In this section, we derive the equations for power of the intermodulation distortion and second harmonic signals generated in the nonlinear acoustic line having a voltage-dependent distributed capacitance $C_{d}(v)$ (12). We assume that the line is driven by two tones $v_{g, \omega_{i}}(t)=\operatorname{Re}\left[V_{g, \omega_{i}} e^{j \omega_{i} t}\right]$ at fundamental frequencies $\omega_{i}$ being $i=1,2$.

If both driving tones are at resonance, their spatial distribution will be that of a standing wave pattern

$$
\begin{aligned}
& v_{\omega_{i}}(x, t)=\operatorname{Re}\left[V_{\omega_{i}} e^{j \omega_{i} t} \cos \left(\frac{\pi x}{l}\right)\right] \\
& i_{\omega_{i}}(x, t)=\operatorname{Re}\left[I_{\omega_{i}} e^{j \omega_{i} t} \sin \left(\frac{\pi x}{l}\right)\right]
\end{aligned}
$$

where the reference $x=0$ is taken at the center of the resonant line of length $l$ (see Fig. 1).

For simplicity, only the first two nonlinear terms of $C_{d}(v)$, i.e., first-order nonlinearities $\left(\Delta C_{1}\right)$ and second-order nonlinearities $\left(\Delta C_{2}\right)$ will be considered. Note however that the procedure could be extended to consider different nonlinear dependences if necessary [17].

The term $\Delta C_{2}$ causes third-order intermodulation products, occurring at $2 \omega_{1}-\omega_{2}$ and $2 \omega_{2}-\omega_{1}$, referred here as IMD3, whereas the nonlinear term $\Delta C_{1}$ produces second-order intermodulation products, at $\omega_{1}+\omega_{2}$ and $\omega_{2}+\omega_{1}$, referred here as IMD2, second harmonic at $2 \omega_{1}$ and $2 \omega_{2}(2 H)$, and also additional contribution to IMD3 due to the mixing of the second harmonic with the fundamental signals.

\section{A. Second-Order Nonlinearities}

Assuming a quadratic nonlinear dependence of the nonlinear distributed capacitance, (12) becomes

$$
\Delta C_{N L}(v)=\Delta C_{2} v^{2} .
$$

We obtain the IMD3 occurring in a nonlinear acoustic transmission line following a similar procedure to that in [17]. Using (20) into (17), we may write the nonlinear differential current as

$$
\frac{\partial i_{n l}(x, t)}{\partial x}=\frac{\partial}{\partial t}\left(\Delta C_{2} v(x, t)^{3}\right)
$$

which can be Fourier transformed to obtain the frequency component at $\omega_{i}$. This results in $\partial I_{n l}(x) / \partial x=$ $j \omega_{i} F\left(\Delta C_{2} v(x, t)^{3}, \omega_{i}\right)$ where $F\left(., \omega_{i}\right)$ indicates the Fourier transform at $\omega_{i}$.

For the third-order intermodulation $2 \omega_{1}-\omega_{2}$ (denoted as $\left.\omega_{12}\right)$, we can write

$$
\frac{\partial I_{n l, \omega_{12}}(x)}{\partial x}=\frac{3}{4} V_{\omega_{1}}^{2} V_{\omega_{2}}^{*}\left[j \omega_{12} \Delta C_{2}\right] \cos ^{3}\left(\frac{\pi x}{l}\right) .
$$


Note that this term might be seen as a current generator distributed along the acoustic line and following a spatial distribution given by the standing-wave pattern at resonance $(\cos (\pi$. $x / l)$ ) affected by the order of the nonlinearities resulting in a term $\cos ^{3}(\pi \cdot x / l)$.

This nonlinear source (22) gives rise to a voltage $V_{\omega_{12}}(x)$, whose spatial distribution should match, except for a multiplicative constant, the one at resonance as in (18). Note that this is true as long as the driving frequencies are both at resonance, i.e., $\omega_{1} \approx \omega_{2} \approx \omega_{12}$.

The voltage $V_{\omega_{12}}(x)$ can be analytically obtained by equating the power generated at $\omega_{12}\left(P_{\text {gen }}\right)$ to the sum of the power dissipated $\left(P_{\text {dis }}\right)$ in the resonator and loads, and $2 \omega_{12}$ times the net reactive stored energy: $P_{g e n}=P_{d i s}+2 j \omega_{12}\left(W_{m}-W_{e}\right)$. Since at resonance $W_{m} \sim W_{e}$, we can write [17]

$$
\begin{aligned}
\frac{1}{2} \int_{-l / 2}^{l / 2} V_{\omega_{12}}(x) d I_{n l, \omega_{12}}^{*}(x) d x \\
=\frac{\omega_{12}}{Q_{L}} \frac{C_{d, 0}}{2} \int_{-l / 2}^{l / 2} V_{\omega_{12}}(x) V_{\omega_{12}}^{*}(x) d x
\end{aligned}
$$

where $Q_{L}$ is the loaded quality factor. The voltage at $\omega_{12}$ can be written as $V_{\omega_{12}}(x)=V_{\omega_{12}} g_{v}(x)$ where $g_{v}(x)$ describes its spatial dependence normalized to a maximum value, and $V_{\omega_{12}}$ is the magnitude to obtain.

Define the normalized stored energy in the resonator $W_{0, v}$ as a function of the maximum voltage $V_{\omega_{12}}$ as

$$
W_{0, v}=\frac{C_{d}}{2} \int_{-l / 2}^{l / 2} g_{v}(x) d x
$$

and the spatial coefficient $\Gamma_{\omega_{12}}$ as

$$
\Gamma_{\omega_{12}}=\frac{1}{2} \int_{-l / 2}^{l / 2} \cos ^{3}\left(\frac{\pi x}{l}\right) g_{v}(x) d x .
$$

We may substitute (24) and (25) into (23) to obtain $V_{\omega_{12}}$

$$
V_{\omega_{12}}=-j \frac{3}{4} \frac{Q_{L}}{W_{0, V}} \Gamma_{\omega_{12}} V_{\omega_{1}}^{2} V_{\omega_{2}}^{*} \Delta C_{2} .
$$

Using the assumption $\omega_{1} \approx \omega_{2} \approx \omega_{12}$, i.e., $g_{v}(x)=$ $\cos (\pi x / l)$, the normalized stored energy (24) and the spatial coefficient (25) can be analytically obtained as $W_{0, v}=1 / 4 \cdot C_{d, 0} \cdot l$ and $\Gamma_{\omega_{12}}=3 / 16 \cdot l$, respectively. Then, (26) becomes

$$
V_{\omega_{12}}=-j \frac{9}{16} Q_{L} V_{\omega_{1}}^{2} V_{\omega_{2}}^{*} \frac{\Delta C_{2}}{C_{d, 0}} .
$$

Note that this formulation requires that $\omega_{1}$ and $\omega_{2}$ be at resonance. In fact, if we consider the resonance frequency as the frequency where the current (or voltage) is maximum, this frequency is slightly shifted to higher frequencies than the $\left|S_{21}\right|$ maximum (usually called series resonance) [18]. This is because the acoustic transmission line is loaded by the external components. However, we can assume that both frequencies are close enough to consider $W_{e} \sim W_{m}$ and that $v(x, t)$ follows a standing wave pattern as done in (18).

By using conventional network analysis (Appendix I), we obtain the dissipated power to the load $P_{L, \omega_{12}}$ from the maximum voltage into the acoustic transmission line $V_{\omega_{12}}$ (27). From this analysis, we have derived the power dissipated at the load for

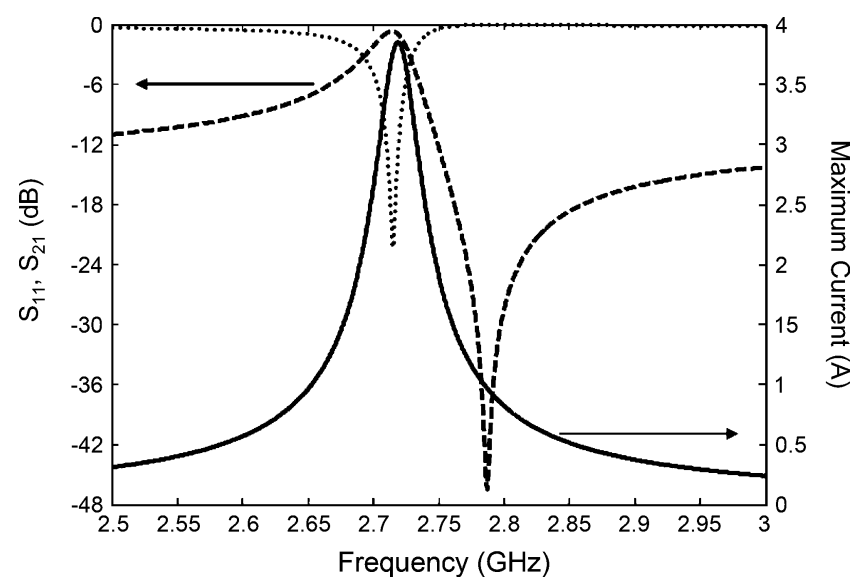

Fig. 3. Left axis: Simulated $S_{21}$ (dashed line) and $S_{11}$ (dotted line) of an AlNFBAR. Right axis: maximum current reached into the resonator (solid line).

TABLE I

COMPARISON OF (27) AND (28) WITH SIMULATIONS

\begin{tabular}{lccc}
\hline \hline & Simulation & $(27)-(28)$ & Error \\
\hline $\operatorname{Mag}\left(V_{\omega l 2}\right)[\mathrm{V}]$ & $6.310^{-4}$ & $6.2310^{-4}$ & $<1.2 \%$ \\
\hline Phase $\left(V_{\omega l 2}\right)$ & $13.9^{\circ}$ & $0.4^{\circ}$ & $13.5^{\circ}$ \\
\hline$P_{L, \omega l 2}[\mathrm{dBm}]$ & -48.5 & -48.5 & $<1 \%$ \\
\hline \hline
\end{tabular}

the case of a two-port FBAR with $50 \Omega$ at the source and load ports

$$
P_{L, \omega_{12}}=36 P_{0, \omega_{1}}^{2} P_{0, \omega_{2}}\left(\frac{Q_{L}}{\omega_{12} W_{0}} \frac{\beta}{1+2 \beta}\right)^{4}\left(\omega_{12} \Delta C_{2}\right)^{2} \Gamma_{\omega_{12}}^{2}
$$

where $P_{0, \omega 1}$ and $P_{0, \omega 2}$ are the input power at $\omega_{1}$ and $\omega_{2}$, respectively, and $\beta$ is the external coupling coefficient defined as the ratio between the dissipated power at one of the external loads and the dissipated power inside the resonator. This coupling coefficient can be calculated from the scattering parameters as $\beta=\left|S_{21}\right|_{m} / 2\left(1-\left|S_{21}\right|_{m}\right)$, being $\left|S_{21}\right|_{m}$ the maximum in the frequency response of $\left|S_{21}\right|$.

Equations (27) and (28) have been validated by performing harmonic balance simulations with commercial software [19]. The simulated resonator has $l=2 \mu \mathrm{m}$ of AlN thickness and an area of $A=3500 \mu \mathrm{m}^{2}$. The maximum of $\left|S_{21}\right|$ occurs at 2.7145 $\mathrm{GHz}$ (see Fig. 3), which is slightly shifted down from the frequency that gives maximum current $\left(f_{\max }=2.719 \mathrm{GHz}\right)$ at the ends of the acoustic transmission line $(x=l / 2$ and $x=-l / 2$ in Fig. 1). The unloaded quality factor $Q_{0}$ is set to 970 by loading the ends of the transmission lines with $0.1-\mathrm{m} \Omega$ lumped resistances. The nonlinearities are set to $\Delta C_{2}=10^{-7} \mathrm{~F} /\left(\mathrm{V}^{2} \cdot \mathrm{m}\right)$ which corresponds in order of magnitude to the nonlinear $\Delta c_{2}$ reported in [22]. The 2- $\mu \mathrm{m}$ length of the acoustic transmission line is modeled by 160 nonlinear cells like the one in Fig. 2 (minimum 100 cells per half-wavelength are required to obtained accurate results [17]). The resonator is then fed with two $10-\mathrm{dBm}$ tones spaced $1 \mathrm{kHz}$ and centered at $2.7145 \mathrm{GHz}$. Table I compares the results of the simulations with those from (27) and (28).

From Table I, we see that the agreement is very good although the predicted phase of the voltage differs slightly from the simulated one. If simulations are done at the frequency that gives 


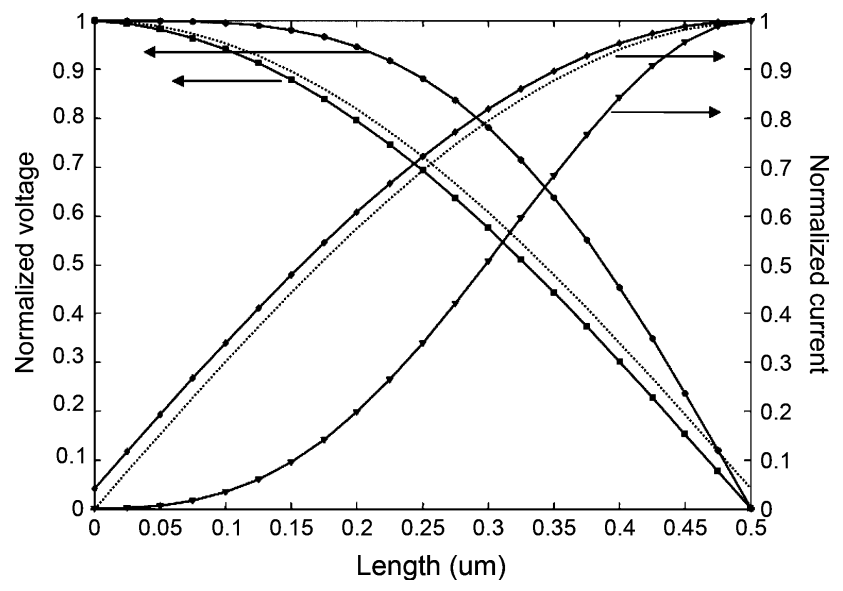

Fig. 4. Voltage (left axis) and current (right axis) distribution in a $1-\mu \mathrm{m}$ length acoustic transmission line from $x=0$ to $l / 2$ for the fundamental signals (squares) and for the $2 \mathrm{H}$ (circles). Dotted line is the distribution corresponding to $\cos (\pi x / l)$ and $\sin (\pi x / l)$.

maximum voltage $\left(f_{\max }=2.719 \mathrm{GHz}\right)$ the phase error is lower than $0.5^{\circ}$. This small error is due to the assumption $W_{e}=W_{m}$ made in our analytical calculations, which does not fully hold in the simulations.

\section{B. First-Order Nonlinearities}

For the analysis of the $2 \mathrm{H}$ and IMD2 generation, we only consider the first term of Taylor's series expansion of $\Delta C_{N L}(v)$

$$
\Delta C_{N L}(v)=\Delta C_{1} v .
$$

The spurious signals occurring at $2 \omega_{1}$ and $\omega_{1}+\omega_{2}$ by (29) are due to the mixing of the fundamentals at $\omega_{1}$ and $\omega_{2}$. On the other hand, the spurious signal at $2 \omega_{1}-\omega_{2}$ is due to the mixing of the second harmonic at $2 \omega_{1}$ with the fundamental at $\omega_{2}$.

1) Second Harmonic and $\omega_{1}+\omega_{2}$ Generation: To obtain the second harmonic, we need to consider the nonlinear current generators at $2 \omega_{1}$ as

$$
\frac{\partial I_{n l, 2 \omega_{1}}(x)}{\partial x}=\frac{1}{2} V_{\omega_{1}}^{2}\left[j 2 \omega_{1} \Delta C_{1}\right] \cos ^{2}\left(\frac{\pi x}{l}\right) .
$$

Unlike in the case described in Section III-A, the nonlinear sources at $2 \omega_{1}$ (30) are weakly coupled to the second resonant mode. This is because the impedance seen at $2 \omega_{1}$ from the center of the acoustic transmission line ( $x=0$ in Fig. 1) to the electrical part of the circuit is very high so the current flowing through the transformer is very small. This high impedance and the symmetrical distribution of the nonlinear sources $\partial I_{n l, 2 \omega_{1}}$ along the line, with a maximum at the center of the acoustic line, forces a zero current at $x=0$ as occurring at the fundamental frequency in the middle of a $\lambda / 2$ line with shorts at both ends. Fig. 4 shows the simulated distribution (from $x=0$ to $x=l / 2$ ) of the voltage and current at $\omega_{1}$ and $2 \omega_{1}$ in the FBAR assessed in Section III-A with $\Delta C_{2}=0$ and $\Delta C_{1} \neq 0$.

The spatial pattern of $V_{2 \omega_{1}}$ results from the combination between the conditions set by the signal generators distributed along the transmission line (30) and the standing wave pattern
TABLE II

VALIDATION OF (31), (33), AND (34) WiTH SimULATIONS

\begin{tabular}{lccc}
\hline \hline & Simulation & $(31),(33),(34)$ & Error \\
\hline $\operatorname{Mag}\left(V_{2 \omega t}\right)[\mathrm{V}]$ & $1.610^{-3}$ & $1.610^{-3}$ & $<0.1 \%$ \\
\hline Phase $\left(V_{2 \omega l}\right)$ & $1.9^{\circ}$ & $1.7^{\circ}$ & $0.2^{\circ}$ \\
\hline $\operatorname{Mag}\left(V_{\omega l+\omega 2}\right)[\mathrm{V}]$ & $3.210^{-3}$ & $3.210^{-3}$ & $<0.2 \%$ \\
\hline Phase $\left(V_{\omega 1+}\right)$ & $1.7^{\circ}$ & $1.7^{\circ}$ & $<0.1^{\circ}$ \\
\hline$P_{2 \omega I}[\mathrm{dBm}]$ & -62.8 & -63.0 & $<0.3 \%$ \\
\hline$P_{\omega 1+\omega 2}[\mathrm{dBm}]$ & -56.8 & -57.0 & $<0.4 \%$ \\
\hline \hline
\end{tabular}

corresponding to $l=\lambda$. Therefore, since the current at $2 \omega_{1}$ is almost zero at the center of the line, the $\partial I_{n l, 2 \omega_{1}}$ generated in a $\partial x$ at $x=0$ flows directly through the distributed capacitance $C_{d, 0}$ (see Fig. 2) generating a voltage drop given by

$$
V_{2 \omega_{1}}=-\frac{1}{2} V_{\omega_{1}}^{2} \frac{\Delta C_{1}}{C_{d, 0}} .
$$

This is the voltage $V_{2 \omega_{1}}$ that directly couples to the electrical circuit part producing measurable second harmonic at the load.

The same procedure is used for the IMD2 at $\omega_{1}+\omega_{2}$ but replacing the $\partial I_{n l, 2 \omega_{1}}$ of (30) by

$$
\frac{\partial I_{n l, \omega_{1}+\omega_{2}}(x)}{\partial x}=V_{\omega_{1}} V_{\omega_{2}}\left[j\left(\omega_{1}+\omega_{2}\right) \Delta C_{1}\right] \cos ^{2}\left(\frac{\pi x}{l}\right)
$$

which implies that

$$
V_{\omega_{1}+\omega_{2}}=-V_{\omega_{1}} V_{\omega_{2}} \frac{\Delta C_{1}}{C_{d, 0}}
$$

that is, the dissipated power at the load at $\omega_{1}+\omega_{2}$ should be 6 $\mathrm{dB}$ greater than that at $2 \omega_{1}$ if the source signals $\omega_{1}$ and $\omega_{2}$ are kept balanced $\left(V_{\omega_{1}}=V_{\omega_{2}}\right)$.

The power coupled to the load is obtained by analyzing the linear circuit at $2 \omega_{1}$ (or $\omega_{1}+\omega_{2}$ adding $6 \mathrm{~dB}$ ) as described in Appendix I, resulting in

$$
P_{L, 2 \omega_{1}}=2 Z_{g}\left(\frac{Q_{L}}{\omega_{1} W_{0, v}} \frac{\beta}{1+2 \beta}\right)^{2} P_{0, \omega_{1}}^{2}\left(\frac{\Delta C_{1}}{C_{d, 0}}\right)^{2} \frac{1}{\left|\phi_{2 \omega_{1}} Z_{2 \omega_{1}}\right|^{2}}
$$

where $Z_{g}$ is the source and load impedance, $\phi_{2 \omega 1}$ is (4) evaluated at $2 \omega_{1}$, and $Z_{2 \omega 1}$ is given by (48). The normalized energy will be $W_{0, v}=1 / 4 C_{d, 0} l$.

Expressions (31), (33), and (34) have been validated by performing harmonic balance simulations [19], using the FBAR described in Section III-A, where the nonlinear terms are now $\Delta C_{2}=0$ and $\Delta C_{1}=10^{-5} \mathrm{~F} /(\mathrm{V} \cdot \mathrm{m})$. Comparison between simulated results and closed-form expressions are summarized in Table II, showing very good agreement.

2) Third-Order Intermodulation Product $2 \omega 1-\omega 2$ : The results of mixing the spurious signal at $2 \omega_{1}$ (and $2 \omega_{2}$ ) with the fundamental signal at $\omega_{2}$ (and $\omega_{1}$ ) give rise to IMD3 at $2 \omega_{1}-\omega_{2}$ (and $2 \omega_{2}-\omega_{1}$ ), both within the resonance band of the FBAR structure. We have checked that no other components like $\omega_{1}-\omega_{2}$ contribute significantly to the IMD3 since the voltages reached inside the resonator at these low frequencies are very low. 
The equation for the third-order intermodulation distortion produced by a first-order nonlinear effect can be derived following steps similar to those used in Section III-A. In this case, the nonlinear sources are

$$
\frac{\partial I_{n l, \omega_{12}}(x)}{\partial x}=j \omega_{12} \Delta C_{1} V_{2 \omega_{1}}(x) V_{\omega_{2}}^{*} \cos \left(\frac{\pi x}{l}\right)
$$

where $V_{2 \omega_{1}}(x)$ is a standing wave pattern described by a function $f_{2 \omega_{1}}(x)$ which resembles a cosine function as shown in Fig. 4. Its maximum value $V_{2 \omega_{1}}$ is given by (31).

As done in Section III-A, we equate the power generated at $\omega_{12}$ with the power dissipated using (23). This results in

$$
V_{\omega_{12}}=j \frac{1}{2} \frac{Q_{L}}{W_{0}} \Gamma_{\omega_{12}}^{\prime} V_{\omega_{1}}^{2} V_{\omega_{2}}^{*} \frac{\left(\Delta C_{1}\right)^{2}}{C_{d, 0}}
$$

where $\Gamma_{\omega_{12}}^{\prime}=1 / 2 \int_{-l / 2}^{l / 2} f_{2 \omega_{1}}(x) \cos ^{2}(\pi x / l) d x$ and $W_{0}=$ $1 / 4 C_{d, 0} \cdot l$.

We can also write this equation as

$$
V_{\omega_{12}}=j \gamma_{\omega_{12}} \frac{4}{3 \pi} Q_{L} V_{1}^{2} V_{2}^{*}\left(\frac{\Delta C_{1}}{C_{d}}\right)^{2}
$$

where the factor $\gamma_{\omega_{12}}$ is defined by

$$
\gamma_{\omega_{12}}=\frac{\Gamma_{\omega_{12}}^{\prime}}{\frac{1}{2} \int_{-l / 2}^{l / 2} \cos ^{3}\left(\frac{\pi x}{l}\right) d x} .
$$

The term $\gamma_{\omega_{12}}$ quantifies the error assuming that the second harmonic follows the standing-wave pattern at $2 \omega_{1}$, i.e., $f_{2 \omega_{1}}(x)=\cos (\pi x / l)$ (see Fig. 4). We have numerically evaluated (38) for several examples being $\gamma_{\omega_{12}}$ always less than 1.15. Therefore, the error will be within $15 \%$ if the approximation $f_{2 \omega_{1}}(x)=\cos (\pi x / l)$ is used to avoid numerical procedures.

Again we are interested in obtaining the dissipated power to the load. In this case, we can write (see Appendix I)

$$
\begin{aligned}
P_{L, \omega_{12}}=64 P_{0, \omega_{1}}^{2} P_{0, \omega_{2}} & \left(\frac{Q_{L}}{\omega_{12} W_{0, v}} \frac{\beta}{1+2 \beta}\right)^{4} \\
& \times\left(\omega_{12} \Delta C_{1}\right)^{2} \Gamma_{\omega_{12}}^{\prime 2}\left(\frac{\Delta C_{1}}{2 C_{d, 0}}\right)^{2} .
\end{aligned}
$$

It is remarkable to note that the power of the IMD3 generated by a nonlinear model of first-order (39) can be larger than the measured power $2 \mathrm{H}$ or IMD2 (34). For example, the ratio between maximum voltages at IMD3 and $2 \mathrm{H}$ results in

$$
\left|\frac{V_{\omega_{12}}}{V_{2 \omega_{1}}}\right|=\gamma_{\omega_{12}} \frac{8}{3 \pi} Q_{L}\left|V_{\omega 2}\right|\left(\frac{\Delta C_{1}}{C_{d}}\right) .
$$

From (40), we see that the ratio between IMD3 and $2 \mathrm{H}$ is proportional to the fundamental signal $V_{2 \omega_{1}}$, the nonlinear coefficient $\Delta C_{1}$, and the loaded quality factor. This is because, unlike $2 \mathrm{H}$ signals, the IMD3 spurious signals are at resonance, so their amplitude is affected by the quality factor of the resonator.

Again we use circuit simulations [19] to validate (37) and (39). Table III summarizes the comparison between simulated
TABLE III

VALIDATION OF (37) AND (39) With SimUlations

\begin{tabular}{lccc}
\hline \hline & Simulation & $(37),(39)$ & Error \\
\hline $\operatorname{Mag}\left(V_{a / 2}\right)[\mathrm{V}]$ & $7.4810^{-4}$ & $7.3610^{-4}$ & $<1.7 \%$ \\
\hline Phase $\left(V_{\omega l 2}\right)$ & $-165.2^{\circ}$ & $-179.1^{\circ}$ & $<14^{\circ}$ \\
\hline$P_{L, \omega l 2}[\mathrm{dBm}]$ & -47.1 & -47.0 & $<0.2 \%$ \\
\hline \hline
\end{tabular}

values and the values obtained analytically, showing good agreement. In this case, $\gamma_{\omega_{12}}$ (38) has also been numerically solved resulting in $\gamma_{\omega_{12}}=1.11$, therefore $\Gamma_{\omega_{12}}^{\prime}=1.11 \cdot 2 / 3 \pi \cdot l$.

Note than the IMD3 is $10 \mathrm{~dB}$ higher than the IMD2 (see Table II) despite the fact that we are only considering nonlinear effects due to first-order nonlinearities.

\section{EXPERIMENTAL RESULTS}

The following section uses the circuit model and the closedform expressions presented in Sections II and III to extract the nonlinear material parameters of a BAW resonator from measurements of the spurious signals at IMD3, IMD2, and 2H.

\section{A. Test Device}

The FBAR resonator tested has a 1- $\mu \mathrm{m}$-thick aluminum nitride (AIN) membrane having an area of $4000 \mu \mathrm{m}^{2}$. The electrodes are made of titanium (30 nm thick) and platinum (150 $\mathrm{nm}$ thick). All materials are deposited onto a silicon substrate. Fabrication details are given in [20]. The resulting resonant frequency is around $2.3 \mathrm{GHz}$.

\section{B. Scattering Parameters and Linear Circuit Model}

A preliminary fitting of the linear response (scattering parameters) is required for a proper modeling and characterization of the nonlinear effects occurring in the FBAR presented above. To do that, we measured the scattering parameters from 2 to $6 \mathrm{GHz}$ to include the fundamental resonance and second harmonic frequencies in the analysis. The input power was fixed to $-10 \mathrm{dBm}$ to ensure that the FBAR is operating in linear regime, and we performed on-wafer line-reflect-reflect-match calibrations [21]. Fig. 5 depicts the frequency response of the transmission $\left(S_{21}\right)$ and reflection coefficient $\left(S_{11}\right)$, for a narrow frequency range (from 2.2 to $2.4 \mathrm{GHz}$ ).

The effective electromechanical coupling coefficient is $\kappa=$ $2.2 \%$ and the loaded quality factor is $Q_{L}=60$ at the resonance (maximum $S_{21}$ ), and $Q_{L}=350$ at the antiresonance (minimum $\left.S_{21}\right)$. This low $Q_{L}$ causes high insertion losses which are incremented by the parasitic effects of the pads [20].

Fig. 6 shows the proposed linear model containing parasitic elements to account for the substrate and pads [20]. The FBAR is modeled using the KLM model (Fig. 1) and the acoustic transmission line is divided into 160 cells containing the distributed linear and nonlinear parameters, like that in Fig. 2. The acoustic line is loaded with two transmission line sections at each side modeling the electrodes. These two transmission lines have a significant impact on the FBARs resonance frequency but we will consider that these electrodes do not contribute to the nonlinearities since the titanium and the platinum have a much more linear strain-stress curve than the AlN [22]. For simplicity, each 


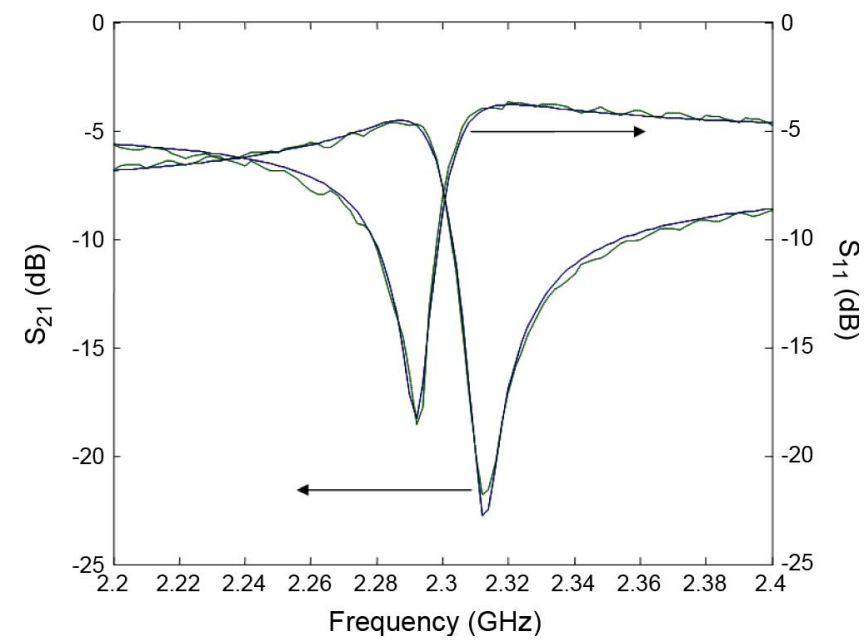

Fig. 5. Simulated (dotted) and measured (solid line) scattering parameters.

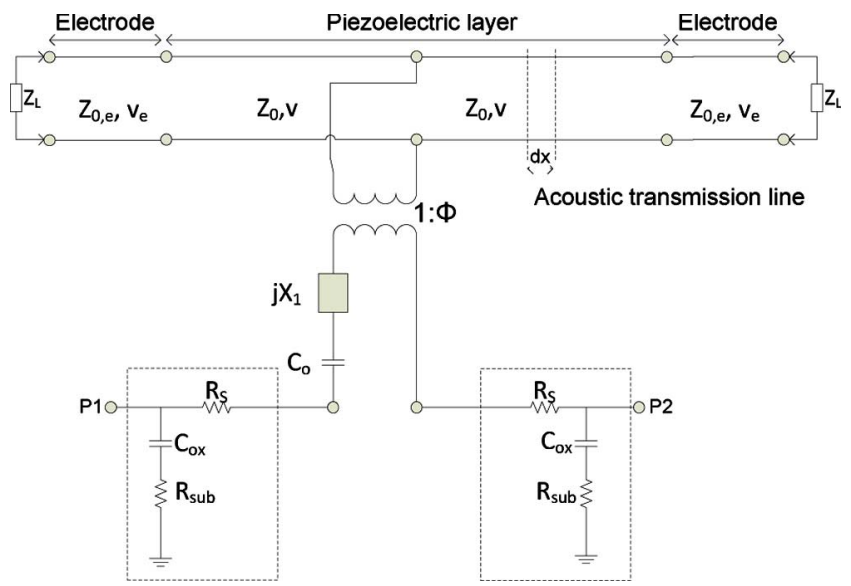

Fig. 6. KLM equivalent circuit including electrodes and parasitic elements $\left(R_{s}, C_{o x}\right.$, and $\left.R_{\mathrm{sub}}\right)$.

180-nm Ti/Pt electrode is modeled as a unique linear transmission line with $v_{p}=3440 \mathrm{~m} / \mathrm{s}$ and $Z_{0}=0.207 \Omega$, which is additionally loaded by a lumped resistor whose value is higher than that of the air impedance, and accounts for acoustic losses.

The values of the circuital components are adjusted by using optimization routines to fit the measured scattering parameters especially at frequencies close to resonance. Fig. 5 shows the frequency response of the simulated circuit model of Fig. 6 and the measurements.

The acoustic losses are modeled with $Z_{L}=4 \cdot 10^{-4} \Omega$ at the ends of the electrodes and the values of the parasitic elements are $R_{s}=9.8 \Omega, R_{\mathrm{sub}}=282 \Omega$ and $C_{o x}=9 \mathrm{pF}$. These parasitic elements are consistent with those extracted from other resonators on the same wafer [11].

\section{Nonlinear Characterization}

To characterize the nonlinear response of our device under test (Fig. 6), we performed intermodulation and second harmonic measurements. For these experiments, we kept both sources balanced in power (i.e., $P_{0, \omega 1}=P_{0, \omega 2}$ ) and swept their powers from -10 to $-10 \mathrm{dBm}$. Both tones $\omega_{1}$ and $\omega_{2}$ are set at resonance with a frequency spacing $\Delta f=f_{2}-f_{1}$

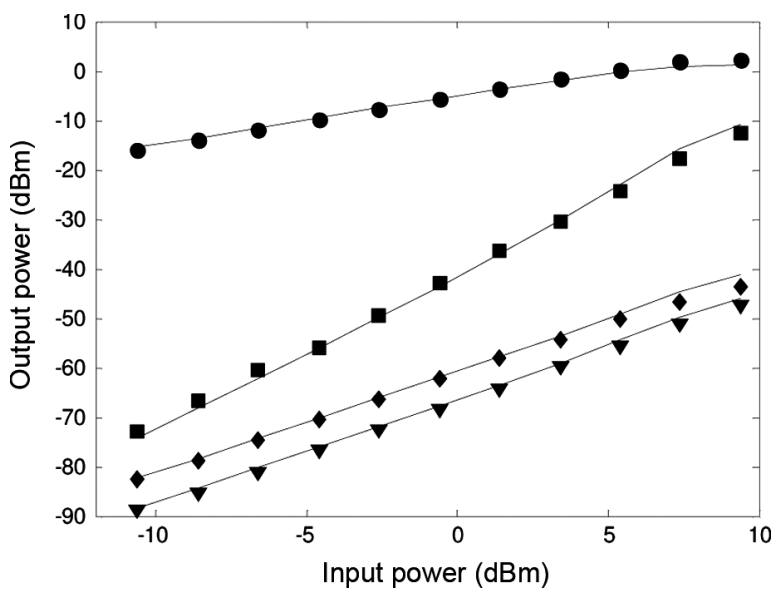

Fig. 7. Measured output power at resonance frequency versus input power for: fundamental power (circles), IMD3 (squares), IMD2 (diamonds), and 2H (triangles). The solid lines represent the simulated results of Section IV-D.

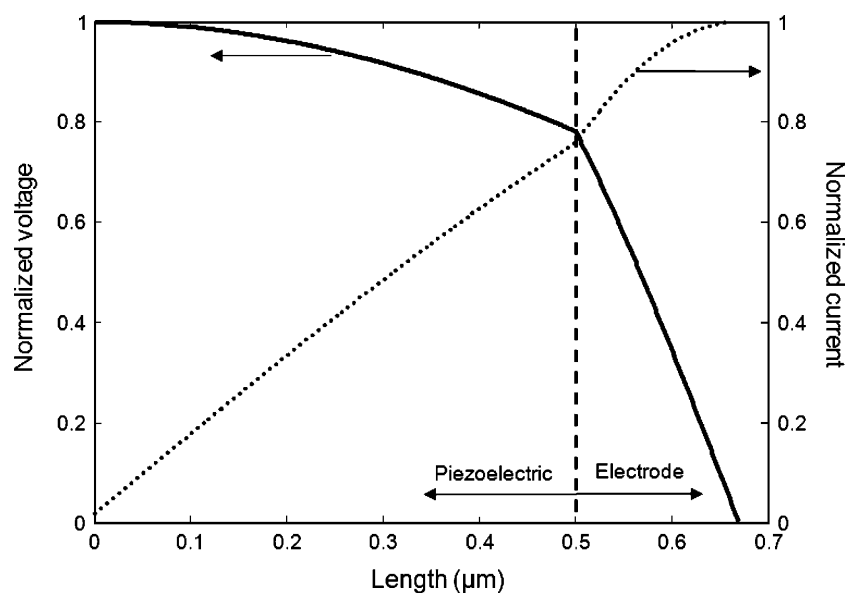

Fig. 8. Normalized current (dotted) and voltage (solid) distribution along the acoustic transmission line: piezoelectric layer and electrode (see Fig. 6).

ranging from $\Delta f=100 \mathrm{~Hz}$ to $\Delta f=1 \mathrm{MHz}$. From these experiments, we obtain the output power of IMD2 $\left(\omega_{1}+\omega_{2}\right)$, IMD3 $\left(2 \omega_{1}-\omega_{2}\right.$ and $\left.2 \omega_{2}-\omega_{1}\right)$, and $2 \mathrm{H}\left(2 \omega_{1}\right.$ and $\left.2 \omega_{2}\right)$ as a function of the input power of the fundamental signal. The dependence of the spurious signals on the fundamental tone frequency spacing $(\Delta f)$ is also evaluated.

Fig. 7 outlines the measured spurious and fundamental signals, when the two fundamental tones are centered at $2.292 \mathrm{GHz}$ (resonance frequency) and $100 \mathrm{~Hz}$ apart. The slope of the fundamental tones is $1: 1 \mathrm{in} \log -\log$ scale up to $+8 \mathrm{dBm}$, indicating that saturation effects do not occur. As one may expect from (28) or (39), and (34), the measured power dependence of IMD3 is $3: 1$ (in $\log -\log$ scale), and $2: 1$ (in $\log -\log$ scale) for $2 \mathrm{H}$ and IMD2.

Next, we extract the nonlinear capacitance $\Delta C(v)$ that fits the measurements. We first extract $\Delta C_{1}$ from the measured $2 \mathrm{H}$ and IMD2 experiments using (34); we evaluate if the IMD3 is consistent with this $\Delta C_{1}$ using (39), and if not, we obtain $\Delta C_{2}$ using (28).

The parasitic elements will be considered as part of the resonator in order to apply the formulation based on the analysis of 
resonators for the third-order intermodulation. See Appendix II for further details.

1) Second Harmonic $2 H$ and IMD2: We use (34) to obtain the value of $\Delta C_{1}$ that better fits the $2 \mathrm{H}$ dissipated power at the external load $Z_{g}=50 \Omega$. To do that, we first obtain: $\phi_{2 \omega_{1}}=$ 0.59 and $Z_{2} \omega_{1}=123-j 82 \Omega$ using (4) and (51), respectively, from the fitted parameters, $C_{d, 0}=6.44 \cdot 10^{-4} \mathrm{~F} / \mathrm{m}$ from the properties of the material, $\beta=0.665$ using $\beta=\left|S_{21}\right| / 2(1-$ $\left.\left|S_{21}\right|\right)$ [24], and $Q_{L}=58$ using $Q_{L}=\left(\omega_{0} / 2\right) \cdot d \varphi / d \omega_{0}$ [25], where $\varphi$ is the phase of $S_{21}$.

To calculate the normalized energy $W_{0 . v}$, we need to know the standing wave pattern in the acoustic transmission line for the fundamental frequencies. Fig. 8 shows this distribution for the voltage and current along the acoustic line and the electrodes. Since the distributions of Fig. 8 do not follow a cosine function, we need evaluate (24) numerically to obtain $W_{0, v}$ (see Appendix II for more details). This results in $W_{0, v}=1.54 \cdot 1 / 4$. $C_{d, 0} \cdot d_{T}$, that is, a value $54 \%$ larger than the value we would obtain if we would consider an acoustic transmission line of total length $d_{T}$, where $d_{T}$ is the thickness of piezoelectric plus electrodes.

Using (34), we find a value of $\Delta C_{1}$ that fits a measured point of Fig. 7. We obtain $\Delta C_{1}=1.1 \cdot 10^{-5} \mathrm{~F} /(\mathrm{Vm})$. This value also fits the measured IMD2 since the measured value $P_{L, \omega 1+\omega 2}$ is $6 \mathrm{~dB}$ larger than $P_{L, 2 \omega 1}$.

2) IMD3 From First Order Nonlinearities $\left(\Delta C_{1}\right)$ : Applying the value of $\Delta C_{1}=1.1 \cdot 10^{-5} \mathrm{~F} /(\mathrm{Vm})$ obtained above into (39) and using $\Gamma_{\omega_{12}}^{\prime} \sim 2 / 3 \pi \cdot l$, we obtain an IMD3 output power of $P_{L, \omega_{12}} \sim-120 \mathrm{dBm}$, which is much smaller than the measured value. This indicates that the IMD3 cannot be only due to $\Delta C_{1}$ and there must be an additional contribution due to $\Delta C_{2}$.

3) IMD3 From Second-Order Nonlinearities $\left(\Delta c_{2}\right)$ : Now we use (28) to extract $\Delta C_{2}$ from the measured IMD3. In this case, the geometrical factor $\Gamma_{\omega_{12}}$ is calculated using (25) from the voltage shown in Fig. 8. Only nonlinear contributions from the piezoelectric layer are considered, since we assume that the electrodes do not contribute to the nonlinearities. This results in $\Gamma_{\omega_{12}}=2.01 \cdot 3 \cdot d / 16$, where $d$ is the piezoelectric thickness. Doing so we obtain $\Delta C_{2}=2.5 \cdot 10^{-5} \mathrm{~F} /\left(\mathrm{V}^{2} \mathrm{~m}\right)$, and the resulting nonlinear capacitance is $\Delta C_{N L}(v)=1.1 \cdot 10^{-5} v+2.5$. $10^{-5} v^{2}$.

\section{CAD-Based Nonlinear Characterization}

To validate the data obtained from closed-form expressions, we have found $\Delta C_{N L}(v)$ by fitting the measured data to computer-aided design (CAD) simulations of the equivalent circuit [19]. These simulations are not subject to the simplifying assumptions made in the derivation of (28), (34), and (39). Furthermore, they can easily account for changes in the equivalent circuit due to electrodes and other extraneous elements. For example, the equivalent circuit may be easily modified from that in Fig. 1 to the one in Fig. 6 to include the effect of the electrodes.

Using the same input power than in Section IV-C as a fitting point, we obtain $\Delta C_{N L}(v)=1.2 \cdot 10^{-5} v+2.3 \cdot 10^{-5} v^{2}$, which is within a $8 \%$ error in both $\Delta C_{1}$ and $\Delta C_{2}$. This agreement indicates that (28) and (34) are quite general as long as the parasitic effects and electrodes are considered when evaluating some terms used in these equation (see details in Appendix II). Fig. 7

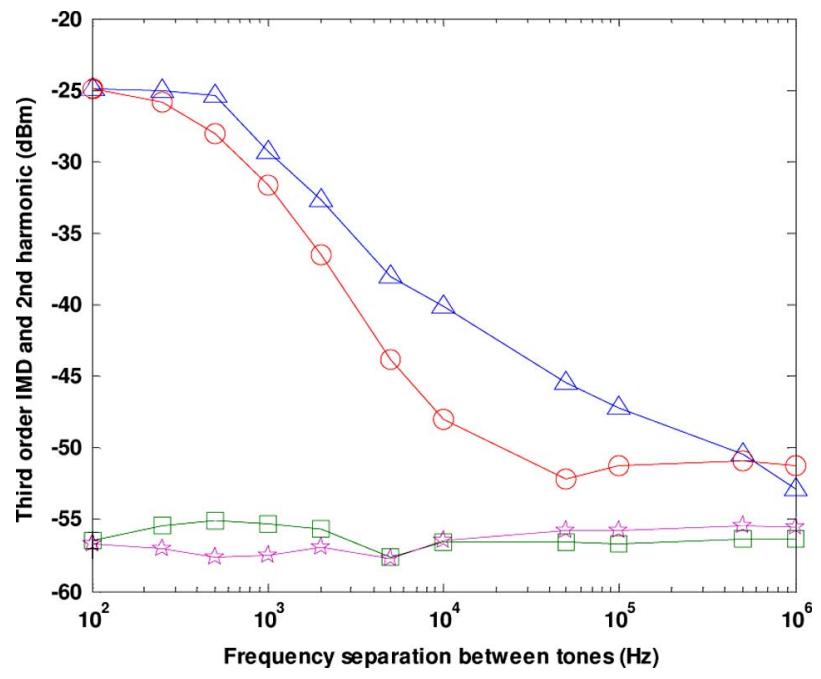

Fig. 9. IMD3 and $2 \mathrm{H}$ output power as a function of the tone spacing $\Delta f$ for an input power of $+5.5 \mathrm{dBm}$. Triangles correspond to $2 \omega_{1}-\omega_{2}$, circles to $2 \omega_{2}-\omega_{1}$, squares to $2 \omega_{1}$, and stars to $2 \omega_{2}$.

shows the measured and simulated results with these extracted nonlinear parameters versus input power.

\section{DISCUSSION AND FURTHER EXPERIMENTS}

The nonlinear coefficients extracted from the measurements $\Delta C_{1}$ and $\Delta C_{2}$ can then be related with the material nonlinear parameter $\Delta c_{N L}^{D}(v)$ through (13) and (14). From (13), we obtain $\Delta C_{1}=1.1 \cdot 10^{-5} \mathrm{~F} /(\mathrm{Vm})$ and the value of the first-order nonlinear elastic stiffness $\Delta c_{1}^{D}=26.5$. This value is six times greater than that reported in [22], which was obtained from the simulations of mechanical displacements.

Using (14) with $\Delta c_{2}=2.3 \cdot 10^{-5} \mathrm{~F} /\left(\mathrm{V}^{2} \mathrm{~m}\right)$, we obtain the second-order nonlinear elastic stiffness $\Delta c_{2}^{D}=$ $-2.1 \cdot 10^{-7} \mathrm{~N}^{-1} \mathrm{~m}^{2}$. This value is three orders of magnitude greater than the one reported in [22]. In fact the value reported in [22] would not be measurable doing IMD experiments since the IMD3 generated by $\Delta C_{1}$ would be greater than that generated by such small $\Delta C_{2}$. The difference between measured and reported results suggests the existence of other causes for the second-order nonlinear effects.

In order to further investigate the nonlinear effects, we performed additional IMD and $2 \mathrm{H}$ measurements versus the frequency spacing between the fundamental tones $\Delta f=f_{2}-f_{1}$. The dependence of IMD power level on the frequency spacing between tones is an indication of dependence with the period of the envelope, and may give additional information to discern between different sources of nonlinear effects.

Fig. 9 depicts the IMD3, IMD2, and $2 \mathrm{H}$ as a function of the frequency spacing for an input power of $+5.5 \mathrm{dBm}$. While $2 \mathrm{H}$ and IMD2 show to be independent of the frequency spacing, that is a constant value of $\Delta c_{1}^{D}$, IMD3 decreases when the frequency spacing increases, which would result in $\Delta c_{2}^{D}$ depending on the frequency spacing between the input tones. This later effect is not considered in the circuit model presented in this paper.

The observed IMD3 dependence on the frequency spacing between the fundamental tones and the difference in the extracted quadratic nonlinear stiffness $\Delta c_{2}^{D}$ is an added indication that 
additional sources may contribute to the second-order nonlinear circuit term $\Delta C_{2} v^{2}$ such as, for example, thermal effects. In addition, Fig. 9 shows asymmetries between the lower and upper $\left(2 \omega_{1}-\omega_{2}\right.$ and $\left.2 \omega_{2}-\omega_{1}\right)$ intermodulation products. This behavior is also characteristic of self-heating effect as shown in [23] which is consistent with the low quality factor of the device. Note that as pointed out before, the stiffened elasticity (10) used in this model fails to predict these thermal effects.

\section{CONCLUSION}

In this paper, we present a novel nonlinear distributed equivalent circuit based on the KLM model. Our new model is useful to account for the linear and nonlinear behavior of BAW resonators in a broad frequency band. We have used it to obtain closed-form expressions for the most relevant nonlinear effects: intermodulation distortion and second harmonic generation. We have checked these expressions with equivalent circuit simulations using harmonic balance techniques.

We have shown that the new equivalent circuit can be easily modified to model the effects of the electrodes and other parasitic elements existing in FBAR. With this modified version of the equivalent circuit, we are able to fit the linear and nonlinear measurements in an FBAR (i.e., s-parameters, intermodulation distortion, and second harmonic generation). The measurements can be fitted to the closed-form expressions for intermodulation distortion and second harmonic generation derived in the paper.

Once the model is fitted to the measurements, we can extract the nonlinear material parameters. The value obtained for $\Delta c_{1}^{D}$ is consistent in order of magnitude with the ones reported in the literature, whereas the $\Delta c_{2}^{D}$ gives a larger value than the ones reported. We suggest that this disagreement on $\Delta c_{2}^{D}$ is because there are other nonlinear effects contributing to the IMD3 generation such as self-heating mechanisms.

The work presented in this paper may now be used in the following aspects.

1) The equivalent circuit could also be used as a basic building block to model more complex devices, such as filters containing several resonators.

2) Evaluation of additional nonlinear effects, such as saturation or detuning, which may also be performed by simulating the equivalent circuit proposed.

3) Inclusion of other nonlinear sources in the model. For example, a nonlinear viscosity could be considered adding a shunted nonlinear distributed conductance to the nonlinear transmission line in the model.

4) Extension of the model to consider self-heating mechanisms.

Although the proposed circuit model has been particularly developed for FBARs with longitudinal propagating wave, this can be generally used for modeling the nonlinear performance in other BAW devices using other propagating modes, for example, quartz crystal resonator operating at the shear mode or even surface acoustic wave resonators, being therefore useful in other applications beyond the scope of this paper.

\section{APPENDIX I \\ IMD AND 2H POWER COUPLED TO THE LOAD}

The power coupled to the load connected at port 2 (Fig. 1) at $\omega_{12}, P_{L, \omega_{12}}$, can be written as

$$
P_{L, \omega_{12}}=\beta P_{r e s, \omega_{12}}
$$

where $\beta=\left|S_{21}\right| / 2\left(1-\left|S_{21}\right|\right)[24]$ and $P_{\text {res }, \omega_{2}}$ is the dissipated power into the resonator. Then, (41) can be written as a function of the unloaded quality factor $Q_{0}$ and the normalized stored energy $W_{0, v}$ as

$$
P_{L, \omega_{12}}=\beta \omega_{12} \frac{W_{0, v}}{Q_{0}}\left|V_{\omega_{12}}\right|^{2}
$$

The dissipated power into the resonator at the fundamental frequency $\omega_{i}$ is given by

$$
P_{r e s, \omega_{i}}=4 P_{0, \omega_{i}} \frac{\beta}{(1+2 \beta)^{2}}
$$

where $P_{0, \omega_{i}}$ is the incident power to the resonator. The maximum voltage at $\omega_{i}$ results in

$$
\left|V_{\omega_{i}}\right|^{2}=4 P_{0, \omega_{i}} \frac{Q_{L}}{\omega_{i} W_{0, v}} \frac{\beta}{(1+2 \beta)} .
$$

For second-order nonlinearities $\left(\Delta C_{2}\right)$, replacing (44) into (26) and the resulting $V_{\omega_{12}}$ into (42), the IMD3 load power gives

$P_{L, \omega_{12}}=36 P_{0, \omega_{1}}^{2} P_{0, \omega_{2}}\left(\frac{Q_{L}}{\omega_{12} W_{0}} \frac{\beta}{1+2 \beta}\right)^{4}\left(\omega_{12} \Delta C_{2}\right)^{2} \Gamma_{\omega_{12}}^{2}$.

The IMD3 coupled to the load for first-order nonlinearities $\left(\Delta C_{1}\right)$ is calculated using (44), (42), and (36)

$$
\begin{aligned}
P_{L, \omega_{12}}=64 P_{0, \omega_{1}}^{2} P_{0, \omega_{2}} & \left(\frac{Q_{L}}{\omega_{12} W_{0, v}} \frac{\beta}{1+2 \beta}\right)^{4} \\
& \times\left(\omega_{12} \Delta C_{2}\right)^{2} \Gamma_{\omega_{12}}^{\prime 2}\left(\frac{\Delta C_{1}}{2 C_{d}}\right)^{2} .
\end{aligned}
$$

Note that this formulation is based on the definition of the quality factor $Q_{L}$ and the coupling coefficient $\beta$ and it does not depend on the origin of the losses. Therefore, it can be used to account for losses modeled as lumped resistances at the ends of the acoustic transmission line, or for acoustic distributed losses which could be modeled adding a series distributed resistance and/or a shunted distributed conductance in the elemental segment of Fig. 2.

The maximum voltage $V_{2 \omega_{1}}$ at the center of the acoustic transmission line is coupled to the electrical part of the circuit through the transformer 1: $\phi$ (see Fig. 1) resulting in a electrical voltage $V_{2 \omega_{1}} / \phi_{2 \omega_{1}}$, thus the current at $2 \omega_{1}$ flowing to the source impedance and load will be

$$
I_{L, 2 \omega_{2}}=\frac{V_{2 \omega_{1}}}{\phi_{2 \omega_{1}}} \frac{1}{Z_{2 \omega_{1}}}
$$

where $\phi_{2 \omega_{1}}$ corresponds to (4) evaluated at $2 \omega_{1}$ and $Z_{2 \omega_{1}}$

$$
Z_{2 \omega_{1}}=2 Z_{g}+j X_{2 \omega_{1}}+\frac{1}{j 2 \omega_{1} C_{0}}
$$


with $X_{2 \omega_{1}}$ obtained from (6) at $2 \omega_{1}$. Therefore, the dissipated power at the load $Z_{g}$ will be

$$
P_{L, 2 \omega_{1}}=\frac{1}{2} Z_{g}\left|V_{2 \omega_{1}}\right|^{2} \frac{1}{\left|\phi_{2 \omega_{1}} Z_{2 \omega_{1}}\right|^{2}}
$$

which, by using (49), (44), and (31), can be written as

$$
P_{L, 2 \omega_{1}}=2 Z_{g}\left(\frac{Q_{L}}{\omega_{1} W_{0, v}} \frac{\beta}{1+2 \beta}\right)^{2} P_{0, \omega_{1}}^{2}\left(\frac{\Delta C_{1}}{C_{d, 0}}\right)^{2} \frac{1}{\left|\phi_{2 \omega_{1}} Z_{2 \omega_{1}}\right|^{2}} .
$$

\section{APPENDIX II \\ PARASITIC AND ELECTRODE EFFECTS}

The parasitic elements may be included as part of the resonator, by using as a quality factor the one obtained from measurements instead of the inherent quality factor of the resonator. The term $Q_{L}(1+2 \beta) / \omega W$ accounts for the acoustic power dissipated in the resonator plus the power dissipated in the parasitic resistances. Note that this does not affect (45) and (46) since the parasitic elements are included in the coupling coefficients and quality factor of the whole device.

However, for the second harmonic power calculation, we need to consider the parasitic elements in the circuit analysis. The electrical voltage $V_{2 \omega_{1}} / \phi_{2 \omega_{1}}$ causes a current $I_{L, 2 \omega_{1}}$ flowing to the load impedance following (47), although in this case, the impedance $Z_{2 \omega_{1}}$ does not follow (48) and it is obtained by conventional circuit analysis of the electrical part of the device including the electrodes (see Fig. 6). This results in

$$
Z_{2 \omega_{1}}=\frac{Z_{g}\left(Z_{g}+Z_{p}\right)}{Z_{g} Z_{p}}\left(2 \frac{Z_{g} Z_{p}}{Z_{g}+Z_{p}}+2 R_{s}+j X_{2 \omega_{1}}+\frac{1}{j 2 \omega_{1} C_{0}}\right)
$$

where $Z_{p}=R_{\text {sub }}+1 /\left(j 2 \omega_{l} C_{o x}\right)$.

In addition, the electrodes may have a significant impact in the spatial distribution of the voltage and current inside the resonator as shown in Fig. 8, so these spatial current and voltage distributions must be considered for the calculation of the normalized energy $W_{0, v}$.

The electrical energy $W_{0, e}$ corresponding to (25) for an ideal acoustic transmission line will be in this case

$W_{0, e}=\frac{1}{2}\left(C_{d, p} \int_{0}^{d_{p} / 2} g_{v, p}(x) d x+C_{d, e} \int_{d_{p} / 2}^{d_{p} / 2+d_{e}} g_{v, e}(x) d x\right)$

where $C_{d}, g_{v}(x)$ and $d$ are the distributed conductance, normalized voltage distribution and layer thickness, respectively, and the sub index " $p$ " and " $e$ " denote piezoelectric layer and electrode, respectively.

The normalized magnetic energy will be

$$
\begin{aligned}
& W_{0, m}=\frac{1}{2\left|Z_{0, \text { equiv }}\right|^{2}} \\
& \quad \times\left(L_{d, p} \int_{0}^{d_{p} / 2} g_{i, p}(x) d x+L_{d, e} \int_{d_{p} / 2}^{d_{p} / 2+d_{e}} g_{i, e}(x) d x\right)
\end{aligned}
$$

where $g_{i}$ indicates the normalized spatial current distribution. $Z_{0 \text {,equiv }}$ is the ratio of maximum voltage and maximum current in a acoustic line loaded with electrodes. This constant can be calculated using conventional microwave analysis of the loaded transmission lines

$$
Z_{0, \text { equiv }}=j\left(Z_{0, e} \sin \varphi_{e} \cos \varphi_{p}+Z_{0, p} \cos \varphi_{e} \sin \varphi_{p}\right)
$$

where $Z_{0}$ and $\varphi$ are the characteristic impedance and electrical length, respectively.

The normalized energy $W_{0, v}$ will be then

$$
W_{0, v}=W_{0, e}+W_{0, m}
$$

\section{ACKNOWLEDGMENT}

The authors would like to thank H. Campanella from the Centre National de Microelectrónica (CNM-CSIC) for providing the FBAR used in this work.

\section{REFERENCES}

[1] K. M. Lakin, "Thin film resonator technology," IEEE Trans. Ultrason. Ferroelectr. Freq. Control, vol. 52, no. 5, pp. 707-716, May 2005.

[2] M. Planat and D. Hauden, "Nonlinear properties of bulk and surface acoustic waves in piezoelectric crystals," Ferroelectrics, vol. 42, pp. 117-136, 1982.

[3] D. A. Hall, "Nonlinearity in piezoelectric ceramics," J. Mater. Sci., vol. 36, pp. 4575-4601, 2001.

[4] H. F. Tiersten, "Analysis of intermodulation in rotated Y-cut quartz thickness-shear resonators," in Proc. 28th Annu. Symp. Freq. Control, 1974, pp. 1-4.

[5] J. Nosek and L. Burianova, "About nonlinear effects in the quartz homeotypes single crystals," in Proc. IEEE Freq. Control Symp., May 2008, pp. 586-591.

[6] J. Nosek, "A precise measurement of some nonlinear effects and its application to the evaluation of nonlinear elastic constants of quartz and GaPO4," Rev. Sci. Instrum., vol. 68, no. 8, pp. 3143-3149, Aug. 1997.

[7] L. Dworsky and R. G. Kinsman, "A simple single model for quartz crystal resonator low level drive sensitivity and monolithic filter intermodulation," IEEE Trans. Ultrason. Ferroelectr. Freq. Control, vol. 41, no. 2, pp. 261-268, Mar. 1994.

[8] R. Aigner, N.-H. Huynh, M. Handtmann, and S. Marksteiner, "Behavior of BAW devices at high power levels," in IEEE MTT-S Int. Microw. Symp. Dig., Jun. 2005, pp. 12-17.

[9] M. Ueda, M. Iwaki, T. Nishihara, Y. Satoh, and K.-Y. Hashimoto, "A circuit model for nonlinear simulation of radio-frequency filters using bulk acoustic wave resonators," IEEE Trans. Ultrason. Ferroelectr. Freq. Control, vol. 55, no. 4, pp. 849-856, Apr. 2008.

[10] K. S. Van Dyke, "The piezo-electric resonator and its equivalent network," Proc. IRE, vol. 16, no. 6, pp. 742-764, Jun. 1928.

[11] E. Rocas, C. Collado, J. Mateu, H. Campanella, and J. M. O'Callaghan, "Third order intermodulation distortion in film bulk acoustic resonators at resonance and antiresonance," in IEEE MTT-S Int. Microw. Symp. Dig., Jun. 2008, pp. 1259-1262.

[12] Y. Cho and J. Wakita, "Nonlinear equivalent circuits of acoustic devices," in Proc. IEEE Ultrason. Symp., Nov. 1993, vol. 2, pp. 867-872.

[13] E. Rocas, C. Collado, A. Padilla, J. Mateu, and J. M. O'Callaghan, "Nonlinear distributed model for IMD prediction in BAW resonators," in Proc. IEEE Int. Ultrason. Symp., Nov. 2008, pp. 1557-1560.

[14] R. Krimholtz, D. A. Leedom, and G. L. Matthaei, "New equivalent circuits for elementary piezoelectric transducers," Electron. Lett., vol. 6, no. 13, pp. 398-399, Jun. 1970.

[15] B. A. Auld, Acoustic Fields and Waves in Solids. Malabar, FL: Krieger, 1990, vol. I.

[16] J. Enlund, V. Yantchev, and I. Katardjiev, "4E-6 electric field sensitivity of thin film resonators based on piezoelectric AlN thin films," in Proc. IEEE Int. Ultrason. Symp., Oct. 2006, pp. 468-471.

[17] C. Collado, J. Mateu, and J. M. O'Callaghan, "Analysis and simulation of the effects of distributed nonlinearities in microwave superconducting devices," IEEE Trans. Appl. Supercond., vol. 15, no. 1, pp. 26-39, Mar. 2005.

[18] T. F. Hueter and R. H. Bolt, Sonics: Techniques for the Use of Sound and Ultrasound in Engineering and Science. New York: Wiley, 1955. 
[19] Agilent Technologies, Advanced Design System, 2006 [Online]. Available: www.agilent.com

[20] H. Campanella, P. Nouet, A. Uranga, P. de Paco, N. Barniol, and J. Esteve, "Automated on-wafer extraction of equivalent-circuit parameters in thin-film bulk acoustic wave resonators and substrate," Microw. Opt. Technol. Lett., vol. 50, no. 1, pp. 4-7, 2008.

[21] F. Purroy and L. Pradell, "New theoretical analysis of the LRRM calibration technique for vector network analyzers," IEEE Trans. Instrum. Meas., vol. 50, no. 5, pp. 1307-1314, Oct. 2001.

[22] S. P. Lepkowski and G. Jurczak, "Nonlinear elasticity in III-N compounds: Ab initio calculations," Phys. Rev. B, Condens. Matter, vol. 72, 2005, 245201 .

[23] J. R. Wilkerson, K. G. Gard, A. G. Schuchinsky, and M. B. Steer, "Electro-thermal theory of intermodulation distortion in lossy microwave components," IEEE Trans. Microw. Theory Tech., vol. 56, no. 12, pp. 2717-2725, Dec. 2008.

[24] M. J. Lancaster, Passive Microwave Devices Applications of High Temperature Superconductors. Cambridge, U.K.: Cambridge Univ. Press, 1997.

[25] K. M. Lakin, "Equivalent circuit modeling of stacked crystal filters," in Proc. 35th Annu. Freq. Control Symp., 1981, pp. 257-262.

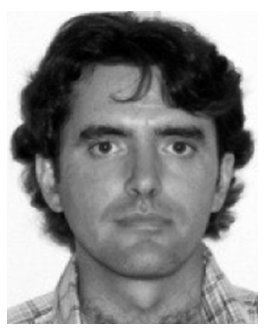

Carlos Collado (M'03) received the Telecommunication Engineering and Ph.D. degrees from Universitat Politècnica de Catalunya (UPC), Barcelona, Spain, in 1995 and 2001, respectively, and the M.S. degree in biomedical engineering from UPC in 2002.

In 2001, he was awarded a prize for the best doctoral thesis in electronics and telecommunications area from UPC. He joined the faculty at UPC in 1998 and became an Associate Professor in 2005. From November 2005 to January 2008, he was a Vice Dean of the Technical School of Castelldefels (EPSC-UPC), responsible for the telecommunication and aeronautic engineering degrees. He was a visiting researcher at the University of California Irvine in 2004. His primary research interests include microwave devices and systems.

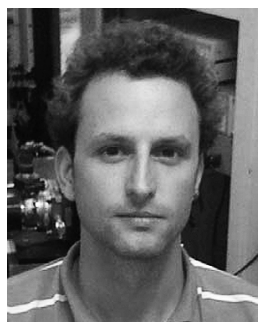

Eduard Rocas ( $\mathrm{S}^{\prime} 07$ ) was born in Palafrugell, Catalonia, Spain, in 1982. He received the Telecommunication Engineering degree from the Universitat Politècnica de Catalunya (UPC), Barcelona, Spain, in 2005, where his final project was associated with the creation of the intelligent communications and avionics for robust unmanned aerial systems (ICARUS) research group. Currently, he is working towards the Ph.D. degree at UPC focusing his research on new materials and structures for novel RF/MW devices.

From September 2005 to July 2006, he was involved in the simulation and modeling of advanced SONARs at the Computer Vision and Robotics Group (VICOROB), University of Girona, as a FPU Grant Holder.

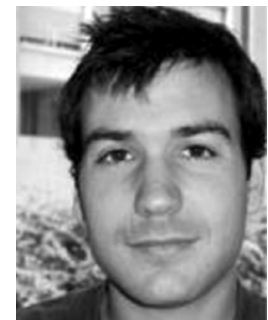

Jordi Mateu (M'03) received the Telecommunication Engineering and Ph.D. degrees from the Universitat Politècnica de Catalunya (UPC), Barcelona, Spain, in 1999 and 2003, respectively.

In 2007, he joined the Department of Signal Theory and Communications, UPC, initially as a Ramon y Cajal Research Fellow and Associate Professor since 2009. He is also a Research Associate with the Centre Tecnològic de Telecomunicacions de Catalunya (CTTC). From May to August 2001, he was a Visiting Researcher with Superconductor Technologies, Inc., Santa Barbara, CA. From October 2002 to August 2005, he was a Research Associate and Coordinator of the communication subsystem area at CTTC, Spain. Since September 2004, he has held several guest researcher appointments at the National Institute of Standards and Technology (NIST), Boulder, CO, where he was a Fulbright Research Fellow from September 2005 to October 2006. From September 2003 to August 2005, he was a part-time Assistant Professor at Universitat Autònoma de Barcelona. He coauthored over 35 papers in international journals, 45 contributions in international conferences, and two book chapters, and holds two patents.

Dr. Mateu was the recipient of the 2004 Prize for the best doctoral thesis in technologies for information and communications by COIT and AEIT. He was also the recipient of a Fulbright Research Fellowship, an Occasional Lecturer Award for visiting MIT, and a Ramón y Cajal Contract.

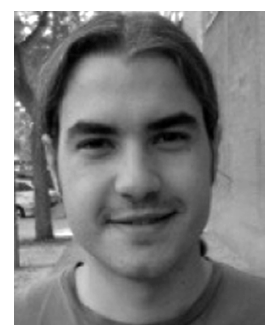

Alberto Padilla (S'09) was born in Barcelona, Spain, in 1984. He received the Telecommunication Engineering degree from the Universitat Politècnica de Catalunya (UPC), Barcelona, Spain, in 2008, where his final project was associated with the mitigation of the nonlinear behavior of high-temperature superconducting planar devices. Currently, he is working towards the Ph.D. degree at UPC, focusing his research on new class of synthesis for microwave filters for satellite communications.

Since March 2008, he has been with the Department of Signal Theory and Communications, UPC, as a Microwave Engineer.

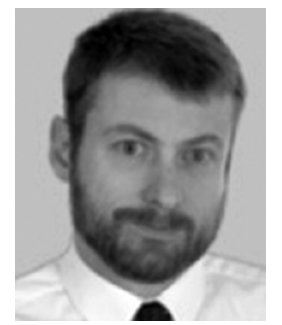

Juan M. O'Callaghan (SM'01) received the Telecommunication Engineering degree from the Universitat Politècnica de Catalunya (UPC), Barcelona, Spain, in 1987, and the M.S. and Ph.D. degrees from the University of Wisconsin, Madison, in 1989 and 1993, respectively.

Currently, he is a Full Professor at UPC. He was an intern at Honeywell's Systems Research Center, Bloomington, MN, working on noise measurement methods for FETs, a Ka band. His research interests include microwave devices and materials and microwave photonics. He has worked on noise characterization, large signal properties of GaAs FETs, and advanced microwave materials such as superconductors and ferroelectrics. He has published over 45 articles in peer-reviewed international magazines and holds three patents. From 2003 to 2006, he served as manager for MERIT, a consortium of European universities delivering a joint Master's program in information technologies within the Erasmus Mundus program. He is currently Vice Dean of Academic Affairs at Telecom BCN, the telecommunication engineering school at UPC. 